\title{
Science Case and Detector Concept for the ARIANNA High Energy Neutrino Telescope at Moore's Bay, Antarctica
}

\author{
Steven W. Barwick ${ }^{a, *}$ on behalf of the ARIANNA Collaboration \\ (a complete list of authors can be found at the end of the proceedings) \\ ${ }^{a}$ University of California, Department of Physics and Astronomy \\ Irvine, California, 92617, USA \\ E-mail: sbarwick@uci.edu
}

The ARIANNA neutrino detector, located at sea-level on the Ross Ice Shelf, Antarctica, consists of 200 autonomous and independent detector stations separated by 1 kilometer in a uniform triangular mesh, and serves to inform the planning of the future projects. The primary science mission of ARIANNA is to search for sources of neutrinos with energies greater than $10^{17} \mathrm{eV}$, complementing the reach of IceCube and other neutrino telescopes that focus on lower energies. An ARIANNA observation of a neutrino source would provide strong insight into the enigmatic sources of cosmic rays. ARIANNA observes the radio emission from high energy neutrino interactions in the Antarctic ice. Among radio based concepts under current investigation, ARIANNA would uniquely survey the vast majority of the southern sky at any instant in time, and an important region of the northern sky, by virtue of its location on the surface of the Ross Ice Shelf at Moore's Bay. The broad sky coverage is specific to the Moore's Bay site, providing capabilities to observe explosive sources from unknown directions. The ARIANNA architecture is designed to measure the angular direction to within 4 degrees for every neutrino candidate, which too plays an important role in the pursuit of multi-messenger observations of astrophysical sources. The sea level location reduces the impact of a potentially serious background associated with the cores of cosmic ray air showers striking the ice surface, generating radio pulses which are similar to those expected from neutrino events. Reflecting layers at the bottom or within the ice sheet (which are known to exist in thick ice sheets) might create a troublesome rate of background events, provided the reflection coefficients are large enough, that arrive at the detector from the same directions as neutrinos.

$37^{\text {th }}$ International Cosmic Ray Conference (ICRC 2021)

July 12th - 23rd, 2021

Online - Berlin, Germany

\footnotetext{
*Presenter
} 


\section{Introduction}

The ARIANNA high energy neutrino detector[1], located at sea-level on the Ross Ice Shelf in a coastal location of Antarctica known as Moore's Bay, consists of 200 autonomous and independent detector stations separated by 1 kilometer in a uniform triangular mesh. As a consequence of the reflection properties at the ice-water interface at the bottom of the Ross Ice Shelf, ARIANNA views almost the entire southern sky, including the galactic center, with nearly uniform exposure. ARIANNA (Figure 1 Left) exceeds the instantaneous sky coverage of all other radio-based neutrino detectors being studied. It's broad sky coverage and reconstruction capabilities of the neutrino direction are well suited to contribute to multi-messenger campaigns initiated by gravitational-wave detectors, gamma-ray telescopes, cosmic ray observatories, and neutrino telescopes targeting lower energies, such as IceCube in the Southern hemisphere, and KM3Net, Baikal-GVD, and P-ONE in the Northern Hemisphere. [This paper contains an abbreviated reference list. A more thorough set of references can be obtained from the ARIANNA-200 white paper[1]]

The most secure science argument for ARIANNA is provided by cosmic ray collisions with microwave background photons that produce cosmogenic neutrinos. Apart from the astrophysical neutrinos produced directly at the sources of cosmic rays, cosmogenic neutrinos are produced by the interaction of UHECR protons and nuclei and cosmic microwave photons These interactions typically occur close to the source, and the neutrino preserves the cosmic-ray direction. Thus, cosmogenic neutrinos can reveal the sources of cosmic rays, though they have not been detected so far. In 10 years of operation, ARIANNA will be sensitive to cosmogenic fluxes at a level of $E_{v}^{2} \Phi \leq$ $4 \times 10^{-9} \mathrm{GeVcm}^{-2} \mathrm{~s}^{-1} \mathrm{sr}^{-1}$, corresponding to $\sim 10 \%$ of the current limits for neutrino energies above $10^{18} \mathrm{eV}$. The observation or upper limit from ARIANNA will constrain model parameters, such as source evolution, energy cutoff and cosmic ray composition.

With a combined fit to the energy spectrum and $X_{\max }$ distribution (an estimator of the cosmicray mass) of UHECR data, the parameters of cosmic-ray sources are estimated from which the cosmogenic neutrino flux can be predicted. However, the analysis is based on a number of simplified assumptions (e.g. a continuous distribution of identical sources and rigidity dependent maximum energies) and the results possess large uncertainties. For example, analysis of the data of the Pierre Auger Observatory located in Argentina finds substantial differences to an analysis of the data of the Telescope Array (TA) located in Utah [2]. The former favors a heavy composition with a low rigidity cutoff at the source resulting in a small cosmogenic neutrino flux, whereas the latter favors a high rigidity cutoff and a slightly lighter source composition resulting in a much higher neutrino flux. Furthermore, data of the Pierre Auger Observatory is compatible with an additional proton contribution resulting in substantial increase in the expected neutrino flux [3].

We summarize the different predictions of cosmogenic neutrinos as well as the predicted ARIANNA sensitivity, and results from existing experiments in Fig. 1 (right). The prediction from TA data is well within the reach of ARIANNA. For the more pessimistic source parameters derived from Auger data, ARIANNA may observe cosmogenic neutrinos if the proton fraction is larger than $20 \%$ of the total particle number. Thus, ARIANNA will provide new insights into the properties of cosmic-ray sources.

To conclude, cosmogenic neutrino detection provides the rationale for the size of the ARIANNA array. A more pragmatic reason is that design costs and the development of institutional 

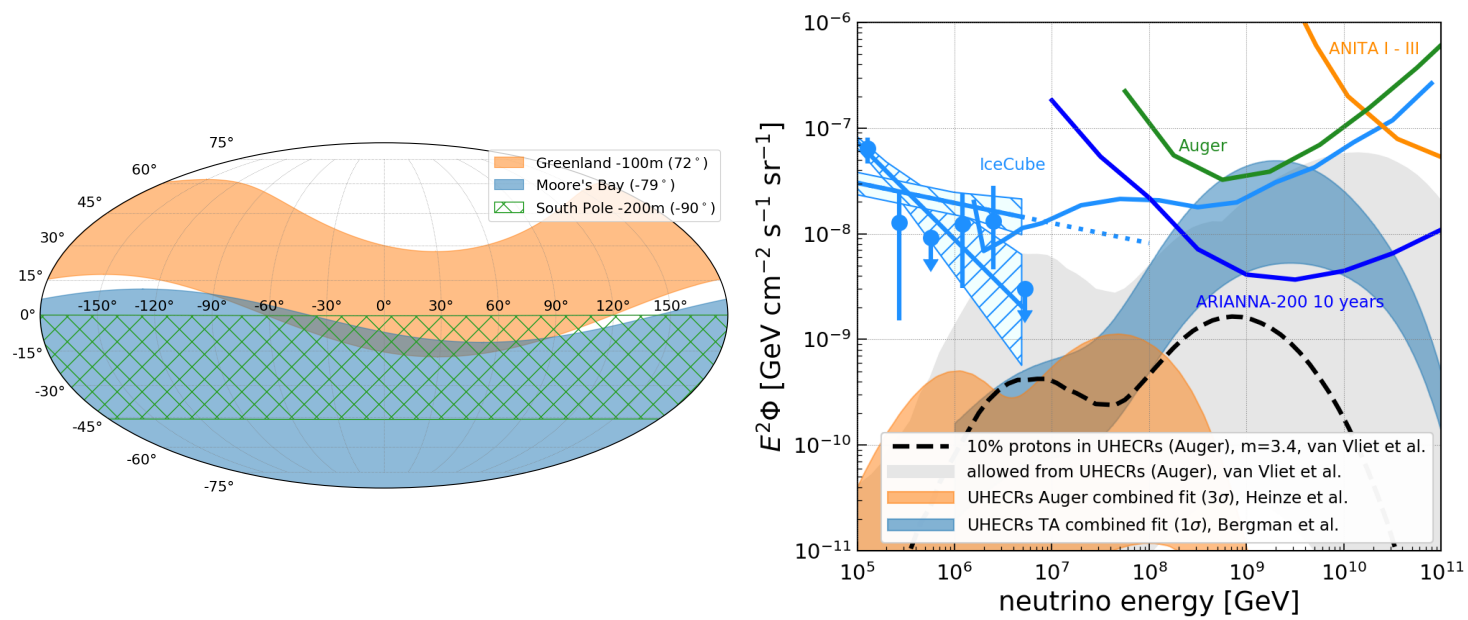

Figure 1: Left: Instantaneous sky coverage of an array of 200 ARIANNA stations at Moore's Bay, Antarctica (Blue), plotted in Right Ascension (RA) and Declination (Dec) at one particular time of the day. For comparison, the sky coverage is shown for radio-based neutrino detectors located at Summit Station in Greenland (gold) and South Pole, Antarctica (green hatch). Right: Expected sensitivity of the ARIANNA detector of 200 stations in one-decade energy bins calculated using NuRadioMC [4] for 10 years of operation assuming a run time of $100 \%$ of a calendar year. Also shown is the measured astrophysical neutrino flux from IceCube limits from existing experiments (solid curves) The color shaded bands show predictions using a simple astrophysical model with commonly discussed source evolution parameters based on cosmic ray data of the Telescope Array (blue) and the Pierre Auger Observatory (orange). The dashed line shows a slightly more complex model with an additional small proton component. The gray band indicates the range of theoretical uncertainties on model parameters [for references, see [1]].

infrastructure to assemble and certify the construction of 200 stations imposes fixed costs that do not scale linearly with station number. For example, reducing the station design from 200 station to 100 stations saves only about $25 \%$ of the project costs.

The sky coverage of ARIANNA augments the point source capabilities of IceCube. At high neutrino energies $\left(E_{v}>\sim 10^{14} \mathrm{eV}\right)$, the Earth becomes opaque. Thus, at higher energies, both IceCube and ARIANNA observe mostly the Southern sky, leading to a substantial overlap in sky coverage. Figure 2 examines the relative sensitivity as a function of energy for an explosive or flaring source at an arbitrary declination of $-23^{\circ}$ in the Southern sky. The strong second minimum at $10^{18} \mathrm{eV}$ (solid curve, right panel) indicates that ARIANNA will observe about one event for every three source events of the highest energy neutrinos observed by IceCube $\left(>10^{14.5} \mathrm{eV}\right)$, assuming neutrino production above $10^{15} \mathrm{eV}$ with an unbroken power law up to $10^{20} \mathrm{eV}$ proportional to $E_{v}^{-2}$. A spatially coincident detection of the same source would establish a hard spectrum up to an energy of $10^{18} \mathrm{eV}$ or greater, and provides evidence for a non-homogeneous distribution the accelerators of the very highest energy cosmic rays.

The model parameter-space for neutrino fluxes of sources is large. It is quite possible that new experimental results will be able to guide theory in this respect. 

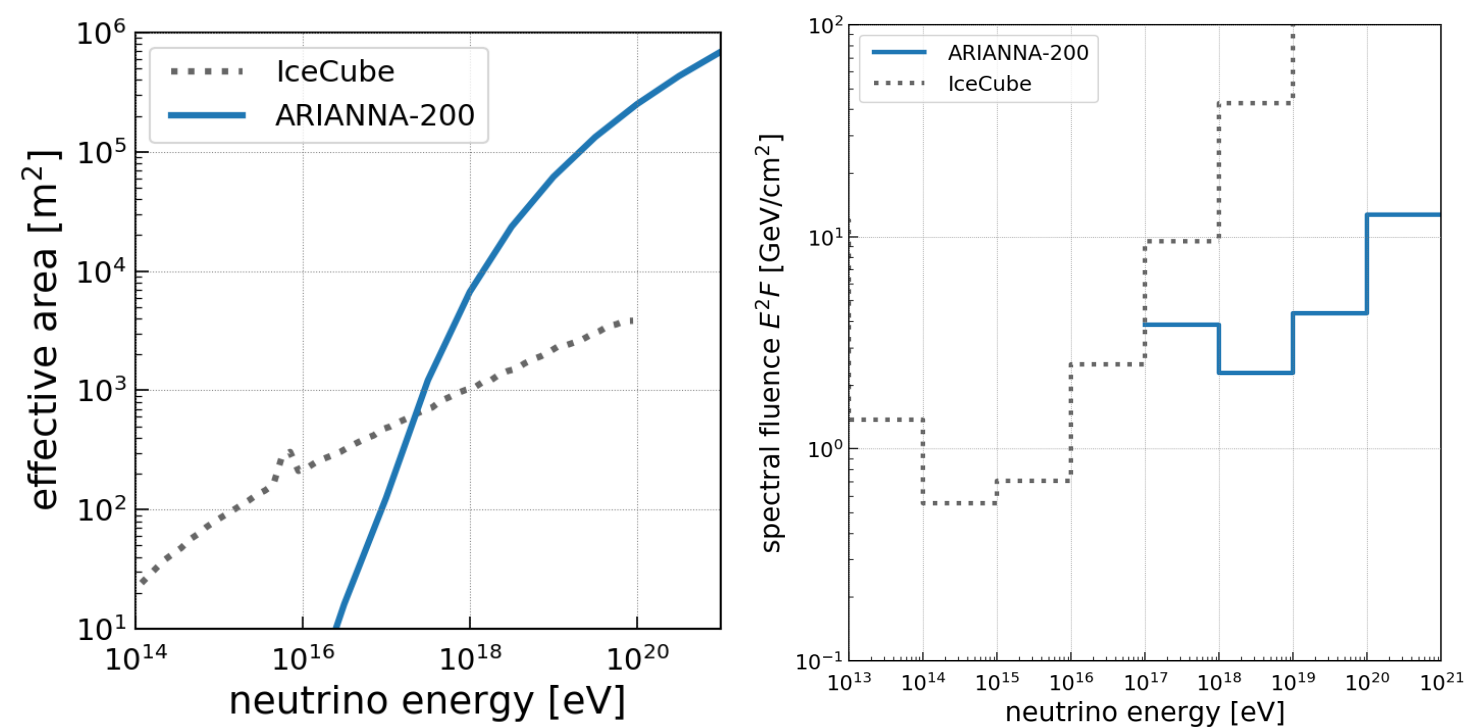

Figure 2: (left) Comparison of effective area for an array of 200 ARIANNA stations and IceCube as a function of neutrino energy, averaged over neutrino flavor and averaged over the sky. (right) Comparison of point fluence sensitivity at $90 \%$ C.L. for the ARIANNA array as in left panel and IceCube as function of energy, both observing a source at declination of -23 degrees.

\section{Description of ARIANNA Detector and Performance}

Similar in basic design to the newer stations in the 10 station test-bed array, each ARIANNA station reads 8 antenna channels: 4 log periodic dipole antennas (LPDA) pointing down, 3 LPDAs pointing up, and a dipole (Fig. 3 left). The LPDAs, which are high-gain broadband receivers, are installed to a depth of only $2 \mathrm{~m}$, while the dipole is located $10 \mathrm{~m}$ below the surface. Based on the ARIANNA test bed experience, there are several options to deploy two hundred ARIANNA stations. In one method, the installation team installs all LPDA antennas in rectangular slots created by electrically heated melting devices, which incorporates the key design elements of the cylindrical hole-melter that successfully drilled several holes to the required depth without the need for continuous supervision.

The performance and reliability of the ARIANNA architecture was also demonstrated by the ARIANNA test bed array, consisting of 7 ARIANNA stations and 3 R\&D stations. It ran successfully from December 2014 to completion of the program in November 2019, achieving operational live-time of $86 \%$ during the sunlit summer months, and a neutrino analysis efficiency of $80 \%$ relative to trigger level.The analysis relied on test bed stations that had neither upward facing LPDA for cosmic ray detection nor a dipole at $10 \mathrm{~m}$. Both antenna systems should further improve the analysis efficiency, as described in the section on Backgrounds. An experimental prototype of a portable wind generator survived for 2 years and achieved 39\% runtime during periods when sunlight was not available. Incremental changes in the geometry of the wind generator and battery capacity are expected to increase the operational live-time to $70 \%$ during the completely dark winter months. To summarize: ARIANNA is expected to operate for more than $80 \%$ of the year using non-centralized fuel-free sources of power.

The ARIANNA test bed array reported a diffuse flux limit of $E_{v}^{2} \Phi \leq 1.7 \times 10^{-6} \mathrm{GeVcm}^{-2} \mathrm{~s}^{-1} \mathrm{sr}^{-1}$, 

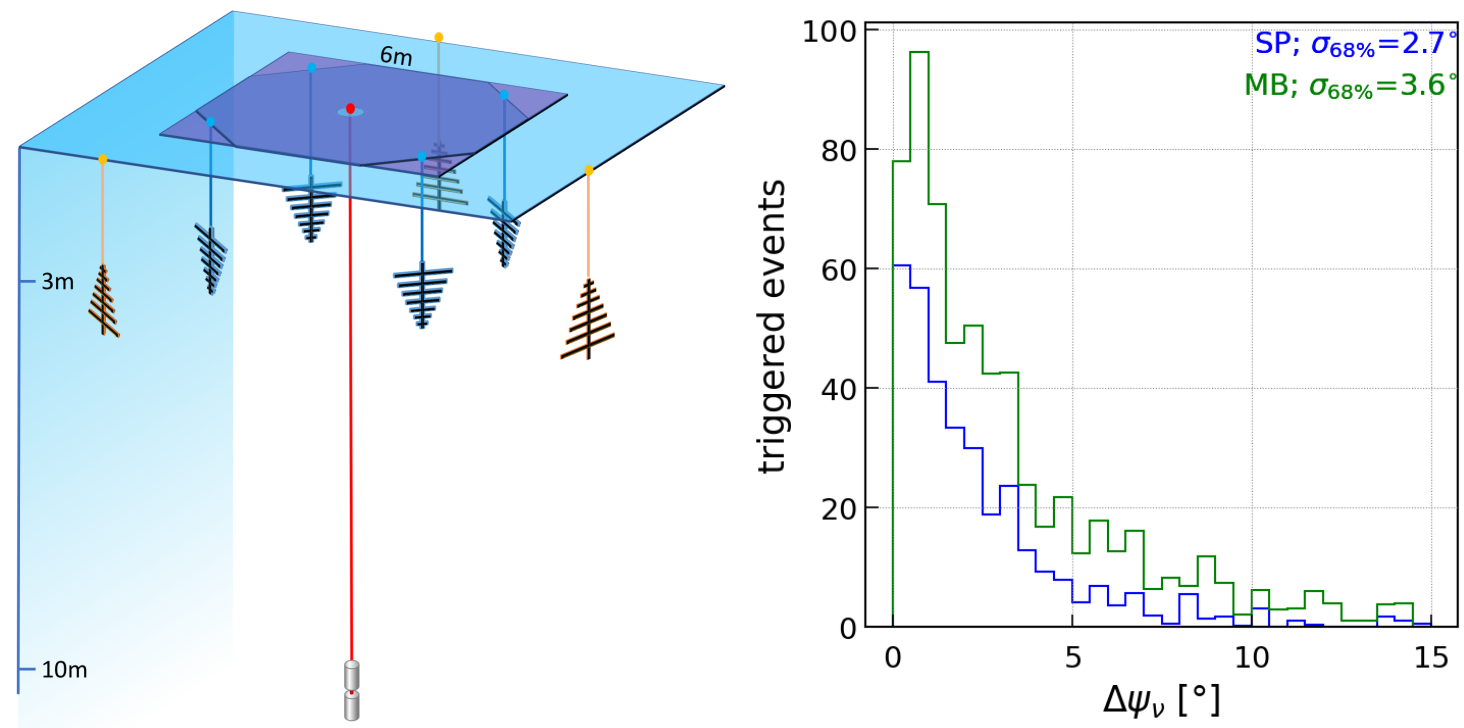

Figure 3: (left) Schematic illustration of one of 200 autonomous, independent stations in the ARIANNA array. All detector components are within $10 \mathrm{~m}$ of the surface. One dipole transmitter for calibration purposes is not shown. (right) Space Angle resolution for all triggered events with $E_{v}=10^{18} \mathrm{eV}$ for detector site at Moore's Bay (MB) and South Pole (SP).

which is a factor 425 larger than the expected sensitivity of ARIANNA. The improved sensitivity in ARIANNA arises from several factors. First the ratio in the number of stations increases by a factor of $200 / 7=28.5$, and we assume the stations operate for 10 years. Since only the first 3 of 7 stations in the test bed ran for 4.5 years and livetime is lost during installation and commissioning, the operational livetime of ARIANNA over 10 years is expected to increase by $10 / 3.5=2.8$. Furthermore, wind generators are assumed to increase the livetime of data collection by a factor 2 due to running over the winter. Realtime rejection of thermal triggers will increase the level 1 trigger rate to from $10 \mathrm{mHz}$ to $100 \mathrm{~Hz}$, and the bandwidth of the trigger will be reduced to optimize the sensitivity [5]. The combination of the last two factors will increase the relative sensitivity by a factor of 2.1. Finally, an assumed analysis efficiency of unity increases the combined improvement to 425 .

ARIANNA achieves this sensitivity by optimizing the trigger bandwidth for the high gain LPDA antennas to reduce the trigger threshold. As discussed in a recent paper [5], the restricted band trigger technique takes advantage of the strong concentration of power at lower frequencies. It indicates that very little signal is lost by restricting the bandwidth to less than $200 \mathrm{MHz}$. However, the RMS noise is reduced significantly so the signal to noise ratio is increased. This leads to a growth in the effective volume of ARIANNA by a factor of almost 3 at neutrino energies near $10^{17} \mathrm{eV}$. Though the trigger bandwidth is reduced, the readout bandwidth of the digitizer cirucit is unrestricted full band. The low frequency edge of the band is produced by a combination of LPDA and amplifier response. To create the upper edge of the frequency band, a 2-pole low pass filter will precede the trigger circuit in the ARIANNA digitizer chip, which is straight-forward to implement.

The ARIANNA station will operate at trigger rates of $100 \mathrm{~Hz}$ to achieve lower thresholds and greater sensitivity. High priority events are saved to disk and transferred by Iridium satellite to 
computer facilities at UC Irvine. These events are identified by a newly developed convolutional neural network (CNN), which we have implemented on the data acquisition system of the ARIANNA test bed station (see A.Anker, these proceedings).

The angular direction of the neutrino is computed from the polarization angle (recently measured by the ARIANNA station to an accuracy of $1^{\circ}$ statistical, $2.7^{\circ}$ systematic [6]), the arrival direction at the detector and the viewing angle relative the Cherenkov cone. The viewing angle measurement benefits from the large bandwidth of the LPDAs and data acquisition electronics. Using NuRadioMC simulation tools developed by the ARIANNA collaboration [4], a full reconstruction of neutrino direction from captured waveforms was developed[7]. The space angle resolution is shown in right panel of Fig. 3. ARIANNA will measure the angular direction of neutrino event at trigger level with an accuracy of $3.6^{\circ}$. The estimated angular resolution is comparable to the systematic error obtained from the in-situ pulser [6] tests and perhaps slightly better than the resolution of high energy cascade events in IceCube, complementing its search for point sources. The resolution for an ARIANNA surface station at the South Pole is better because more events are detected with nearly vertical polarization, resulting in stronger signals in the dipole. Neutrino energy requires a measurement of the distance to the interaction vertex, which is measured by the powerful DnR technique, to identify the location of the vertex of nearly every event [8] with high precision. In the DnR method, the distance to the vertex is related to time difference between two signal paths of the radio emission, one that propagates directly to the dipole receiver and the second ray that reflects from the surface to the dipole. Many systematic uncertainties are reduced by observing the time delay in a single dipole. Consequently, ARIANNA test bed studies have shown that the relative precision of the time delay is $<0.1 \mathrm{~ns}$. ARIANNA will measure the cascade energy to within $25 \%$, consistent with expectation[7] [9].

\section{Backgrounds}

Since the goal of the ARIANNA project is to increase the sensitivity by a factor of 500 , rare backgrounds must be evaluated and mitigated. We consider both anthropogenic and physics backgrounds due to cosmic-ray interactions. The protected geography of Moore's Bay shields ARIANNA from anthropogenic radio-frequency noise created by McMurdo Station[10]. The analysis efficiency was not impacted by known anthropogenic sources [11]. Backgrounds associated with radio production by cosmic-ray collisions in the atmosphere are intrinsically directional and they can be identified by upward facing LPDA antennas [12]. In addition, atmospheric cosmic ray signals and other radio noise propagating downward will not produce the characteristic double pulse waveform in the dipole antenna employed by the DnR method for neutrino vertex reconstruction [8]. Background events correlated with high winds, presumably created by static discharge from drifting snow, provided the the most challenging background observed by the ARIANNA test-bed stations[11]. These backgrounds will be strongly suppressed by the multi-pulse characteristics of the neutrino signal in the dipole, the downward direction from the time delay between the surface LPDA and the dipole, and the up/down amplitude asymmetry in the LPDA. Due to these improvement in the design relative to the primary station design of the pilot array, the analysis efficiency is expected to increase to near unity. 
Radio pulses generated in the atmosphere by cosmic rays may travel through the ice, reflect from the bottom, and travel upward to the ARIANNA station. Though the rate of these events have not been calculated yet, the station has the capability to identify these events. The DnR technique will accurately reconstruct the distance to the source of radio emission (essentially, the propagation distance to shower max) to values much greater than a few $\mathrm{km}$, precluding a vertex within the ice. For many reflected events, the dipole will observe little or no signal, which is also incompatible with the expectation from neutrino interactions. We note that an accurate distance measurement relies on the accurate characterization of the reflective properties from the ice-water interface, where much is known at the Moore's Bay site. Also, the frequency content in cosmic ray signals is peaked a lower frequencies than typical of neutrinos, and further modified by the maximally-long pathlength of travel through the ice. For cosmic ray directions with large zenith angles, the reflected signals may be tagged by the detection of the downward traveling radio signal by the upward pointng LPDA antennas on each station.

There are two additional backgrounds associated with cosmic rays. One background arises from high-energy muons in cosmic ray air showers that penetrate the ice surface and occasionally radiate high energy photons within the ice. The photons initiate an electromagnetic shower in the vicinity of the ARIANNA station that appears identical to a neutrino signal. The expected rate of background events for the full ARIANNA array is less than 0.05 events per year for $E_{s h}>10^{17} \mathrm{eV}$ [13]. Thus, for the sensitivity of this project, the rate of muon background events is small even after 10 years of operation.

Another potential background is generated by the cores of cosmic ray air showers that strike the snow surface, producing an Askaryan signal within the first $10 \mathrm{~m}$ in the ice near the surface[14][15]. If the core-induced radio signal is then reflected into the upward traveling direction by the reflecting layer (for ARIANNA site, the reflecting layer is the water-ice boundary; at higher elevations, it will likely be internal reflecting layers), it will be difficult to distinguish from neutrino signals by direction. Fortunately, the fractional energy of most cores that survive to sea-level is small and the core radius is larger than at higher elevation sites, so fewer particles in the residual shower participate in the coherent radio production. The ARIANNA station will identify this background using several of the same techniques mentioned earlier. The combination of a well-studied [16] specular ice-water reflector at Moore's Bay and the DnR technique will position the vertex at the surface with about $10 \%$ uncertainty in the depth, or approximately $110 \mathrm{~m}$, where few neutrino interactions occur except at the highest energies.

The ARIANNA array provides redundant modes of cosmic-ray detection. The relatively narrow separation of $1 \mathrm{~km}$ between stations provides high efficiency detection of cosmic rays arriving with large zenith angles, but more vertical directions are detected with significantly less efficiency due to the decreasing footprint of the annular radio ellipse on the surface. For these directions, possible detection is provided by scattered power in the upper firn, followed by horizontal propagation to a nearby station. It is reported that $\sim 1 \%$ of the emitted electric field is trapped by density fluctuations in the ice strata near the surface. Once captured, the radio pulse may propagate horizontally, with a $400 \mathrm{~m}$ attenuation length [17]. The ARIANNA project is large enough to observe enough events to calibrate the efficiency of this CR tagging strategy. 


\section{Why Now?}

ARIANNA, an intermediate scale project of 200 surface stations, will search for astrophysical and cosmogenic neutrinos at energies beyond the reach of IceCube with a factor 10 improvement in sensitivity over current limits. ARIANNA may be the first to observe a diffuse flux of neutrinos created by the GZK mechanism. Viewing half the sky for explosive or flaring emission is unique to the Moore's Bay site. The detection of emission from a source would present significant new opportunities. The architecture is sufficiently mature to take the next step. A measurement of the cosmogenic neutrino flux in the early years of operation will inform the science community on how to optimize the experimental efforts to extract insights on the nature of the cosmic-ray accelerators. In addition, ARIANNA will quantify the rate of rare, difficult to predict, or unexpected background processes. The development and validation of background mitigation strategies is imperative for the successful operation of future, large scale, radio-based neutrino detectors.

\section{References}

[1] A. Anker et al. arXiv:2004.09841 (2020) .

[2] D. Bergman for the Telescope Array Collaboration Proc. 36th ICRC 2019, Madison, Wisconsin, USA, PoS(ICRC2019)190 .

[3] A. van Vliet, R. A. Batista, and J. R. Hörandel Phys. Rev. D 100 no. 2, (July, 2019) 021302.

[4] C. Glaser et al. Eur.Phys.J.C 80 (2020) 77.

[5] C. Glaser and S. W. Barwick JINST 16 no. 05, (May, 2021) T05001.

[6] ARIANNA Collaboration, A. Anker et al. JINST 15 no. 09, (2020) P09039.

[7] G. Gaswint, "Ph.d dissertation, University of California-Irvine," 2021.

[8] ARIANNA Collaboration, A. Anker et al. JCAP 11 (2019) 030.

[9] C. Glaser for the ARIANNA Collaboration Proc. 36th ICRC 2019, Madison, Wisconsin, USA, PoS(ICRC2019)899.

[10] L. Gerhardt et al. Nucl. Inst. Meth. A 624 (2010) 85.

[11] A. Anker et al. JCAP 03 (2020) 053.

[12] S. W. Barwick et al. Astropart. Phys. 90 (2017) 50-68.

[13] D. García-Fernández, C. Glaser, and A. Nelles Phys. Rev. D 102 (2020) 083011.

[14] S. De Kockere, K. de Vries, and N. van Eijndhoven PoS ICRC2021 (talk) 101319.

[15] A. Javaid, "Ph.d dissertation, University of Delaware," 2012.

[16] S. W. Barwick et al. J. Glaciol. 61 no. 227, (2015) 438-446.

[17] R. Lahmann for the ARIANNA collaboration, "Investigations of ice and emitter properties from radio signals recorded with arianna," in Proceedings of the 36th International Cosmic Ray Conference PoS(ICRC2019)939. 2019. 


\section{Full Authors List: ARIANNA Collaboration}

Astrid Anker ${ }^{1}$, Pierre Baldi ${ }^{2}$, Steven W. Barwick ${ }^{1}$, Jakob Beise ${ }^{3}$, Hans Bernhoff ${ }^{4}$, Dave Z. Besson ${ }^{5,6}$, Nils Bingefors ${ }^{3}$, Maddalena Cataldo $^{7}$, Pisin Chen ${ }^{8}$, Daniel García Fernández ${ }^{9}, 7$, Geoffrey Gaswint ${ }^{1}$, Christian Glaser ${ }^{3}$, Allan Hallgren ${ }^{3}$, Steffen Hallmann ${ }^{9}$, Jordan C. Hanson ${ }^{10}$, Spencer R. Klein ${ }^{11}$, Stuart A. Kleinfelder ${ }^{12}$, Robert Lahmann ${ }^{7,1}$, Jiayi Liu ${ }^{1}$, Mitchell Magnuson ${ }^{5}$, Stephen McAleer ${ }^{2}$, Zach Meyers ${ }^{9}$, Jiwoo $\mathrm{Nam}^{8}$, Anna Nelles ${ }^{9,7}$, Alexander Novikov ${ }^{5,6}$, Manuel P. Paul ${ }^{1}$, Christopher Persichilli ${ }^{1}$, Ilse Plaisier ${ }^{9,7}$, Lilly Pyras $^{9}$, Ryan Rice-Smith ${ }^{1}$, Joulien Tatar ${ }^{13}$, Shih-Hao Wang ${ }^{8}$, Christoph Welling ${ }^{9,7}$, Leshan Zhao ${ }^{1}$

${ }^{1}$ Department of Physics and Astronomy, University of California, Irvine, CA 92697, USA.

${ }^{2}$ Department of Information and Computer Science, University of California, Irvine, CA 92697, USA.

${ }^{3}$ Uppsala University Department of Physics and Astronomy, Uppsala SE-752 37, Sweden.

${ }^{4}$ Uppsala University Department of Engineering Sciences, Division of Electricity, Uppsala SE-752 37, Sweden.

${ }^{5}$ Department of Physics and Astronomy, University of Kansas, Lawrence, KS 66045, USA.

${ }^{6}$ National Research Nuclear University MEPhI (Moscow Engineering Physics Institute), Moscow 115409, Russia.

${ }^{7}$ ECAP, Friedrich-Alexander Universität Erlangen-Nürnberg, 91058 Erlangen, Germany.

${ }^{8}$ Department of Physics and Leung Center for Cosmology and Particle Astrophysics, National Taiwan University, Taipei 10617, Taiwan. ${ }^{9}$ DESY, 15738 Zeuthen, Germany.

${ }^{10}$ Whittier College Department of Physics, Whittier, CA 90602, USA.

${ }^{11}$ Lawrence Berkeley National Laboratory, Berkeley, CA 94720, USA.

${ }^{12}$ Department of Electrical Engineering and Computer Science, University of California, Irvine, CA 92697, USA.

${ }^{13}$ Research Cyberinfrastructure Center, University of California, Irvine, CA 92697, USA.

\section{Acknowledgements}

We are grateful to the U.S. National Science Foundation-Office of Polar Programs, the U.S. National Science Foundation-Physics Division (grant NSF-1607719) for supporting the ARIANNA array at Moore's Bay, and NSF grant NRT 1633631. Without the invaluable contributions of the people at McMurdo Station, the ARIANNA stations would have never been built. We acknowledge funding from the German research foundation (DFG) under grants GL 914/1-1 and NE 2031/2-1m, the Taiwan Ministry of Science and Technology, the Swedish Government strategic program Stand Up for Energy, MEPhI Academic Excellence Project (Contract No. 02.a03.21.0005) and the Megagrant 2013 program of Russia, via agreement 14.12.31.0006 from 24.06.2013. 